\title{
Simultaneous Monitoring of the Adsorption and Desorption of Colloidal Particles during Deposition in a Parallel Plate Flow Chamber
}

\author{
J. M. MEINDERS, ${ }^{\prime}$ J. NOORDMANS, AND H. J. BUSSCHER
}

Laboratory for Materia Technica, University of Groningen, Antonius Deusinglaan 1, 9713 AV Groningen, The Netherlands

Received September 20, 1991; accepted December 18, 1991

\begin{abstract}
A technique is described to study simultaneously the adsorption and desorption of colloidal particles on collector surfaces in a parallel plate flow chamber. Using a phase contrast microscope equipped with an ultra long working distance objective, the deposition process can be observed in situ. Analysis of successively stored images yields the time of arrival and departure of each colloidal particle, thus enabling determination of the adsorption and desorption rates, as well as of the total number of particles deposited. In addition the method allows analysis of the spatial arrangement of deposited particles, while distinguishing between upstream and downstream deposition of particles, yielding pair distribution functions, going from $-180^{\circ}$ to $+180^{\circ}$. In this paper the technique has been applied to study the deposition of $736-\mathrm{nm}$ diameter monodisperse polystyrene latex particles from a potassium nitrate solution ( 1 and $60 \mathrm{~m} M$ ) to glass, using varying flow rates $\left(0.034\right.$ to $\left.0.456 \mathrm{~cm}^{3} \cdot \mathrm{s}^{-1}\right)$. Initial deposition rates $j_{0}$ were derived from the total number of adhering particles $n(t)$ as well as from the experimentally measured time dependence of the deposition rate $j(t)$. The results showed clear tendencies with ionic strength and flow rate. The desorption rates $j_{\text {des }}(t)$ were small and the desorption rate coefficients $\beta$ were dependent on the residence times of the adhering particles. The blocked areas, derived from the deposition kinetics, ranged from 8 to 675 times the geometrical cross section of a particle, depending on the experimental conditions.

(c) 1992 Academic Press, Inc.
\end{abstract}

\section{INTRODUCTION}

The deposition of colloidal particles on collector surfaces is of importance in a wide variety of different applications, ranging from carrier flotation, filtration, flocculation, composite material production, fouling of heat exchangers, and thrombus formation in vascular prostheses to microbial adhesion to teeth $(1-7)$.

Thorough understanding of colloidal particle deposition is also important for the proper design of experimental systems to study particle deposition (8) and among the systems used we mention the radial flow chamber (9), the stagnation point flow chamber $(10,11)$, the rotating disk system (12), and the parallel plate flow chamber $(13,14)$.

\footnotetext{
${ }^{1}$ To whom correspondence should be addressed.
}

Studies of colloidal particle deposition involve identification of the factors influencing the kinetics of the process (15-19) and stationary state adhesion (20-22). The results obtained can be used to test the available theories for mass transport governing deposition (23-27). The kinetics of colloidal particle deposition depend, in a complicated way, on the boundary conditions at the collector surface. During the process of coverage by adhering particles, the geometry of the collector surface and the hydrodynamic conditions near the surface change. Thus blocking occurs, resulting in a decreased particle deposition rate on the collector surface. Both blocking and desorption of adhering particles on the collector surface, eventually cause a stationary state to be reached.

Recently both theoretical $(15,28)$ and experimental work $(22,29-31)$ has been carried 
out, analyzing the spatial arrangement of particles adhering to flat collector surfaces. Using image analysis techniques, Sjollema and Busscher (30) described radial and angular distribution functions between polystyrene particles deposited on various collectors in a parallel plate flow chamber, and they showed that an adhering particle can be surrounded by an asymmetrical, blocked zone, in which virtually no particles adhere and which is elongated in the direction of flow. Using these distribution functions, the area blocked by an adhering particle can be effectively estimated. Dabros (28) predicted the existence of such an asymmetrical, blocked shadow zone on the basis of Monte Carlo simulations of the deposition process.

Experimental analysis of the spatial arrangement of adhering particles, as done by Sjollema and Busscher (30), yield radial and angular distribution functions which do not discriminate between upstream and downstream deposition of a particle with respect to an adhering one, i.e., the distribution functions go from $-90^{\circ}$ to $+90^{\circ}$ with respect to the direction of flow. Extension of our computer programs has made it possible to remove this limitation, by storing and analyzing not only the spatial coordinates, but also the time of arrival of an adhering particle (32). Thus a more complete experimental verification of the Monte Carlo simulations by Dabros (28) has become possible.

A slight modification of this extended program also allows simultaneous measurement of adsorption and desorption rates, by comparison and analysis of subsequently grabbed images. Herewith it is possible to quantitatively obtain particle adsorption and desorption rates, their characteristic time dependence, as well as the residence times of adhering particles before desorption.

It is the aim of this paper to present a detailed description of the experimental techniques and computational methods involved in the simultaneous measurement of adsorption and desorption rates. Results are presented for deposition of polystyrene particles onto glass under various conditions and their desorption under the influence of a particle concentration gradient, i.e., when flow was switched to buffer with the same ionic strength but without particles. To enhance the desorption process a surfactant solution was also added. In addition, the distribution functions obtained from these experiments are presented as well.

\section{THEORY}

The most general expression for the deposition rate $j(t)$ of colloidal particles to collector surfaces is given by

$$
j(t)=j_{\text {ads }}(t)-j_{\mathrm{des}}(t),
$$

in which $j_{\text {ads }}(t)$ and $j_{\text {des }}(t)$ are the adsorption and desorption rate, respectively. Stationary state adhesion results both from desorption as well as from blocking (15-27).

The desorption rate $j_{\mathrm{des}}(t)$ can be described as (15)

$$
j_{\mathrm{des}}(t)=\int_{0}^{t} \beta(t-\tau) j(\tau) d \tau,
$$

in which $\beta(t-\tau)$ is the desorption rate coefficient, where $\tau$ is the time at which a particle was adsorbed, and $t$ is the time at which a particle desorbs. Note that $(t-\tau)$ is the residence time of an adhering particle.

Exact forms of $\beta(t-\tau)$ are not known, but it seems reasonable to propose that $\beta(t-\tau)$ changes exponentially from some initial value $\beta_{0}$ to a final value $\beta_{\infty}$ with a relaxation time $1 / \delta$, i.e.,

$$
\beta(t-\tau)=\beta_{\infty}-\left(\beta_{\infty}-\beta_{0}\right) e^{-\delta(t-\tau)} .
$$

Provided that $j_{\text {ads }}(t)$ is constant and equals the initial deposition rate $j_{0}$ and that $\beta(t-\tau)$ does not depend on the number of adhering particles, it can be shown (15) from Eqs. [1][3] that

$$
\begin{aligned}
j(t)=\frac{j_{0}}{\Delta}\left[p_{1} e^{p_{1} t}-p_{2} e^{p_{2} t}\right. & \\
& \left.+\delta\left(e^{p_{1} t}-e^{p_{2} t}\right)\right]
\end{aligned}
$$


and

$$
\begin{aligned}
n(t)=\frac{j_{0}}{\Delta}\left[\left(1+\frac{\delta}{p_{1}}\right) e^{p_{1} t}\right. & -\left(1+\frac{\delta}{p_{2}}\right) e^{p_{2} t} \\
& \left.-\left(\frac{\delta}{p_{1}}+\frac{\delta}{p_{2}}\right)\right],
\end{aligned}
$$

where

$$
p_{1,2}=\frac{1}{2}\left[-\left(\beta_{0}-\delta\right) \pm \Delta\right]
$$

and

$$
\Delta=\left[\left(\delta+\beta_{0}\right)^{2}-4 \beta_{\infty} \delta\right]^{1 / 2} .
$$

There are some special cases of Eq. [3] which we want to point out here:

$$
\text { (1) } \beta(\mathrm{t}-\tau)=\beta_{0}
$$

This special case indicates that the probability of desorption is constant in time and independent of the residence time of an adhering particle. For this case (15)

$$
j(t)=j_{0} e^{-\beta_{0} t}
$$

and

$$
\begin{gathered}
n(t)=\frac{j_{0}}{\beta_{0}}\left(1-e^{-\beta_{0} t}\right) . \\
\text { (2) } \beta_{\infty} \approx 0 .
\end{gathered}
$$

This special case indicates that the probability of desorption becomes negligible when a particle has been residing on the surface long enough, i.e., interaction forces become stronger in time. It can be shown (15) that

$$
j(t)=\frac{j_{0}}{\delta+\beta_{0}}\left(\delta+\beta_{0} e^{-\left(\delta+\beta_{0}\right) t}\right),
$$

and the number of particles adhering on the collector surface will be

$$
\begin{aligned}
n(t)= & \frac{j_{0}}{\delta+\beta_{0}} \\
& \times\left[\delta t+\frac{\beta_{0}}{\delta+\beta_{0}}\left(e^{-\left(\delta+\beta_{0}\right) t}+1\right)\right] .
\end{aligned}
$$

(3) $\beta_{\infty}>\beta_{0}$.
This special case indicates that the probability of desorption increases when a particle has been adhering longer on the surface, i.e., the interaction forces become weaker in time. Under such conditions the effective particle deposition rate becomes a damped oscillating function and the number of adhering particles varies linearly with time for $\beta_{0} \tau \gg 1$ and reaches a limiting value in an oscillatory fashion.

Dabros and Van de Ven (15) also derived expressions for the adsorption rates of colloidal particles on a collector surface, while neglecting desorption and accounting for blocking, and derived in this approach.

$$
\begin{aligned}
& j(t)=j_{0} e^{-A_{1} j_{0} t} \\
& n(t)=\frac{1}{A_{1}}\left(1-e^{-A_{1} j_{0} t}\right),
\end{aligned}
$$

in which $A_{1}$ is the area blocked by an adhering particle, usually expressed as a factor $\gamma$, according to

$$
A_{1}=\pi a^{2} \gamma
$$

with a the particle radius. Due to hydrodynamic interactions and electrostatic repulsions between adhering particles, $\gamma$ can become as high as $20-30(10)$.

In a more general approach, accounting both for blocking as well as for desorption, we can describe the effective particle deposition rate as

$$
\begin{aligned}
j(t)=j_{0}-A_{1} j_{0} n & \\
& -\int_{0}^{t} \beta(t-\tau) j(\tau) d \tau .
\end{aligned}
$$

Equation [18] can be solved, assuming $\beta(t-$ $\tau)=\beta_{0}$, yielding

$$
j(t)=j_{0} e^{-\left(A_{1} j_{0}+\beta_{0}\right) t}
$$

and

$$
n(t)=\frac{j_{0}}{A_{1} j_{0}+\beta_{0}}\left(1-e^{-\left(A_{1} j_{0}+\beta_{0} t\right)}\right) .
$$

Comparing Eqs. [1], [2], and [18] it can be seen that 


$$
\begin{array}{r}
j_{\text {ads }}(t)=j_{0}-A_{1} j_{0} \int_{0}^{t} j(\tau) d \tau \\
=\frac{j_{0}}{A_{1} j_{0}+\beta_{0}}\left(\beta_{0}+A_{1} j_{0} e^{-\left(A_{1} j_{0}+\beta_{0}\right) t}\right) \\
=j(t)+\beta_{0} n(t)
\end{array}
$$

and that the total number of particles that has ever adhered up until time $t$ equals

$$
\begin{aligned}
& n_{\mathrm{ads}}(t)=\int_{0}^{t} j_{\mathrm{ads}}(\tau) d \tau \\
&=\frac{j_{0}}{A_{1} j_{0}+\beta_{0}}[ \beta_{0} t+\frac{A_{1} j_{0}}{A_{1} j_{0}+\beta_{0}} \\
&\left.\times\left(1-e^{-\left(A_{1} j_{0}+\beta_{0}\right) t}\right)\right] .
\end{aligned}
$$

Analogously, the desorption rate becomes

$$
\begin{array}{r}
j_{\text {des }}(t)=\frac{\beta_{0} j_{0}}{A_{1} j_{0}+\beta_{0}}\left(1-e^{-\left(A_{1} j_{0}+\beta_{0}\right) t}\right) \\
=\beta_{0} n(t)
\end{array}
$$

and the total number of particles that has ever desorbed up until time $t$ equals

$$
\begin{aligned}
& n_{\mathrm{des}}(t)=\int_{0}^{t} j_{\mathrm{des}}(\tau) d \tau=\frac{\beta_{0} j_{0}}{A_{1} j_{0}+\beta_{0}} \\
& \quad \times\left[t-\frac{1}{A_{1} j_{0}+\beta_{0}}\left(1-e^{-\left(A_{1} j_{0}+\beta_{0}\right) t}\right)\right] .
\end{aligned}
$$

\section{MATERIALS AND METHODS}

\section{Polystyrene Particles, Collector Material,} and Experimental Conditions

Monodisperse polystyrene particles (UV148, AKZO Research, Arnhem, the Netherlands) with a diameter of $736 \mathrm{~nm}$ were kindly provided by Dr. R. Zsom, AKZO, the Netherlands. Particles were washed two times by centrifugation in demineralized water and suspended to a concentration of around $3 \times$ $10^{8} \mathrm{~cm}^{-3}$ in a $1 \mathrm{~m} M$ or $60 \mathrm{~m} M$ potassium nitrate $\left(\mathrm{KNO}_{3}\right)$ solution at $\mathrm{pH}$ 7.0. The surface characteristics as well as the preparation of the polystyrene latices have been described by Brouwer and Zsom (33).
Glass plates, used as collector material, were extensively washed with a surfactant RBS in demineralized water, followed by alternately rinsing with methanol and demineralized water. After this cleaning procedure, a zero water contact angle was observed on all plates. The surface characteristics of thus prepared glass plates have been described in detail by Sjollema and Busscher (20).

Experiments were done at different flow rates ranging from 0.034 to $0.456 \mathrm{ml} \cdot \mathrm{s}^{-1}$, corresponding with shear rates ranging from 15 to $200 \mathrm{~s}^{-1}$ yielding Reynolds numbers between 0.9 and 12.0 , well within the range of laminar flow. Enumeration of the number of adhering particles has been done on the bottom plate of the flow chamber.

Six to eight hours after each experiment, flow was switched to potassium nitrate solution without particles in order to monitor desorption stimulated by a concentration gradient. Again after $6-8 \mathrm{~h}$, a small volume of a surfactant solution ( $2 \%$ RBS in demineralized water) was added to enhance desorption. Table I lists the experimental conditions employed for each experimental run with code numbers assigned.

\section{Parallel Plate Flow System and Image Analysis}

The deposition experiments were carried out in a parallel plate flow chamber, which has been extensively described by Sjollema $e t$

TABLE I

\begin{tabular}{lccc}
\multicolumn{4}{c}{$\begin{array}{c}\text { Experimental Conditions Employed for Each } \\
\text { Experimental Run with Code Numbers }\end{array}$} \\
\hline & $\begin{array}{c}\text { Ionic strength } \\
(\mathrm{mM})\end{array}$ & $\begin{array}{c}\text { Particle concentration } \\
\left(\mathrm{cm}^{-3}\right)\end{array}$ & $\begin{array}{r}\text { Flow rate } \\
\left(\mathrm{cm}^{3} \cdot \mathrm{s}^{-1}\right)\end{array}$ \\
Code & 60 & $3.0 \times 10^{8}$ & 0.034 \\
$\lg 127 \mathrm{a}$ & 60 & $3.0 \times 10^{8}$ & 0.114 \\
$\lg 128 \mathrm{a}$ & 60 & $2.8 \times 10^{8}$ & 0.228 \\
$\lg 129 \mathrm{a}$ & 60 & $2.9 \times 10^{8}$ & 0.342 \\
$\lg 130 \mathrm{a}$ & 60 & $2.8 \times 10^{8}$ & 0.456 \\
$\lg 131 \mathrm{a}$ & 1 & $3.3 \times 10^{8}$ & 0.034 \\
$\lg 132 \mathrm{a}$ & 1 & $3.1 \times 10^{8}$ & 0.456 \\
$\lg 133 \mathrm{a}$ & & &
\end{tabular}


al. (34). As an important feature, the flow chamber, with dimensions $16 \times 8 \times 1.8 \mathrm{~cm}$, has a gradually changing inlet and outlet region. This facilitates the establishment of a laminar flow in the center of the chamber (14). The bottom and top plates, with dimensions of $5.5 \times 3.8 \mathrm{~cm}$, are made out of the material to be studied. A Teflon spacer in between the two plates, yields a separation distance of 0.06 $\mathrm{cm}$. A pulse-free flow was created by hydrostatic pressure and the suspension was recirculated by a roller pump.

The entire flow chamber is placed on the stage of a phase contrast microscope (Olympus $\mathrm{BH}-2$ ) equipped with a $40 \times$ objective with an ultra long working distance (Olympus ULWD-CD Plan 40 PL). A CCD camera (CCD-MX High Technology, Eindhoven, The Netherlands) is mounted on the phase contrast microscope and is coupled to an image analyzer (TEA, image-manager, Difa Breda, The Netherlands), installed in a $12 \mathrm{MHz}$ IBM AT personal computer. With this setup, a direct observation of the deposition process in situ is possible without any additional shear forces acting on the deposited particles. Thus the spatial arrangement of deposited particles with respect to each other is fully preserved.

An image is built up out of $512 \times 512$ pixels, with each pixel representing one byte, i.e., 256 gray values ranging from 0 (black) to 255 (white). The magnification of the system is such that the total field of view is $0.017 \mathrm{~mm}^{2}$ and the area of one particle equals approximately 6 pixels.

In order to distinguish between adhering particles and in focus moving particles, two successively ( $\approx 1$-s time interval) grabbed images are multiplied. Because the gray value of the background is substantially higher than the gray value of the particles, this procedure yields higher gray values for moving particles than for adhering particles.

In order to eliminate artifacts caused by dust or dirt on the lenses and camera, a multiplied out-of-focus image is subtracted from the multiplied images, yielding a uniformly gray background. Furthermore, to reduce noise and to amplify the gray value difference between particles and background, a Laplace filter procedure is carried out. Finally the image is thresholded resulting in a black and white image. At this point these images and the times at which they were taken, are written to a hard disk for later analysis. In order to follow the deposition process as closely as possible, images are grabbed and analyzed as described above at the highest possible rate, i.e., with a 110 -s time interval in between.

\section{Data Handling}

The image analysis procedure consisted of a distance transform, separating multiplets into singlets, and converting the particles into single pixels. Thus the spatial coordinates of each particle in every image are known and its time of adsorption and desorption can be obtained by comparison of successively stored images.

The comparison of successively stored images was done by creating so-called "adsorption" and "desorption" images, while making use of the gray values to designate the time of adsorption or desorption of a particle, as schematically illustrated in Fig. 1 and explained below.

The first row designates stored images of adhering particles at different times. The first square in the second row (adsorption image $1-1$ ) is created by giving all particles in the stored image 1 gray value 1 , indicating that these particles have adsorbed before time $t_{1}$. The second square in the same row (adsorption image 1-2) is created by superimposing the stored image 2 on the adsorption image $1-1$. When there is a particle at the same position in the stored image 2 as the adsorption image 1-1, i.e., particle connectivity, the particles remain indexed by gray value 1 . However, particles that have newly deposited between time $t_{1}$ and $t_{2}$ are assigned gray value 2 in the adsorption image $1-2$. Repeating this procedure for every particle and image, we obtain the last adsorption image $1-j$, in which the number of particles with the gray value $i$ 
1

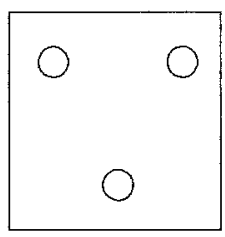

$1-1$

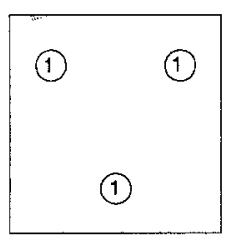

$\uparrow-1$

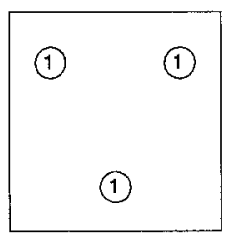

2

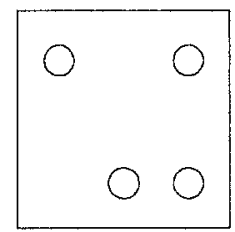

$1+2$



$1-2$

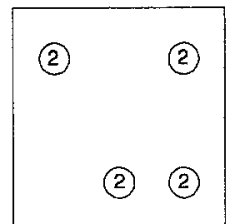

STORED IMAGES
3

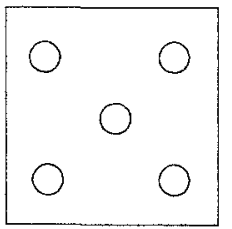

ADSORPTION IMAGES

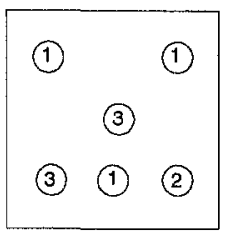

DESORPTION IMAGES

$1-3$

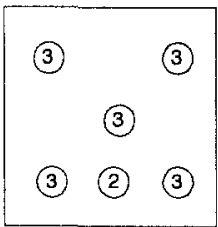

4

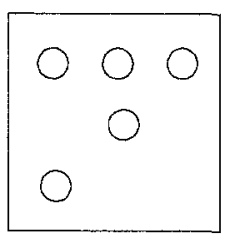

$1-4$

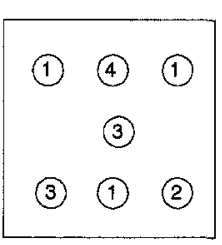

1-4

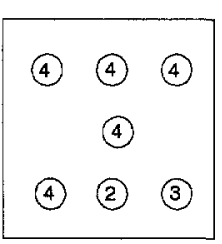

5

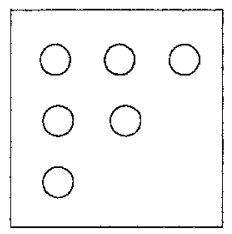

$1-5$

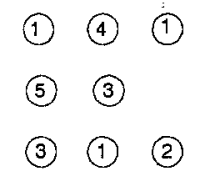

1-5

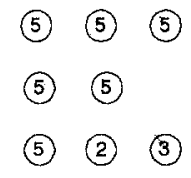

FIG. 1. Illustration of the computational method employed to compare successively stored images by creating so-called "adsorption" and "desorption" images. In an "adsorption" image, particles are given artificial gray values designating the number of the stored image in which they adsorbed. Thus, the gray values in the latest "adsorption" image created indicate when a particle adsorbed on a given position. In a "desorption" image, particles are given artificial gray values designating the number of the stored image in which they desorbed. Thus, the gray values in the latest "desorption" image created indicate when a particle desorbed from a given position.

indicate the number of particles which have been adsorbed between time $t_{i-1}$ and $t_{i}$.

In a similar way, desorption image 1-1 (first square on third row) is created by giving all particles in image 1 gray value 1 , indicating that these particles are adhering in the image taken at time $t_{1}$. The desorption image 1-2 (second square on third row) is created by superimposing the stored image 2 on the desorption image 1-1. Gray values of the particles in the desorption image 1-2 are then made 2, except for those particles in the desorption image 1-1 with no connectivity with particles in the stored image 2. For these particles the grey values remain 1 , indicating de- sorption between time $t_{1}$ and $t_{2}$. From the last desorption image $1-j$, the number of particles with the gray value $i$, provides the number of particles which have desorbed between time $t_{i}$ and $t_{i+1}$.

When these adsorption and desorption images have been created, virtually all parameters described in the theoretical outline of this paper can be derived:

$-j_{\text {ads }}(t)$ follows directly from the adsorption image $1-j$, since division of the number of particles with gray value $i$ in this image, by the time interval between images $i-1$ and $i$ yields the adsorption rate. 
$-j_{\text {des }}(t)$ follows completely analogously from the desorption image $1-j$.

$-j(t)$ can either be obtained directly from the stored images or from

$$
j(t)=j_{\mathrm{ads}}(t)-j_{\mathrm{des}}(t) .
$$$$
n(t)\left[10^{6} \mathrm{~cm}^{-2}\right]
$$

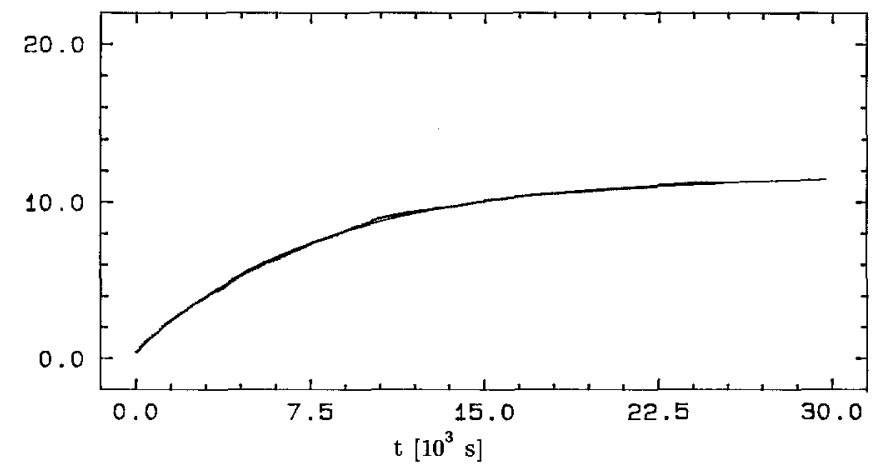

$$
\mathrm{n}_{\text {ads }}(\mathrm{t})\left[10^{6} \mathrm{~cm}^{-2}\right]
$$
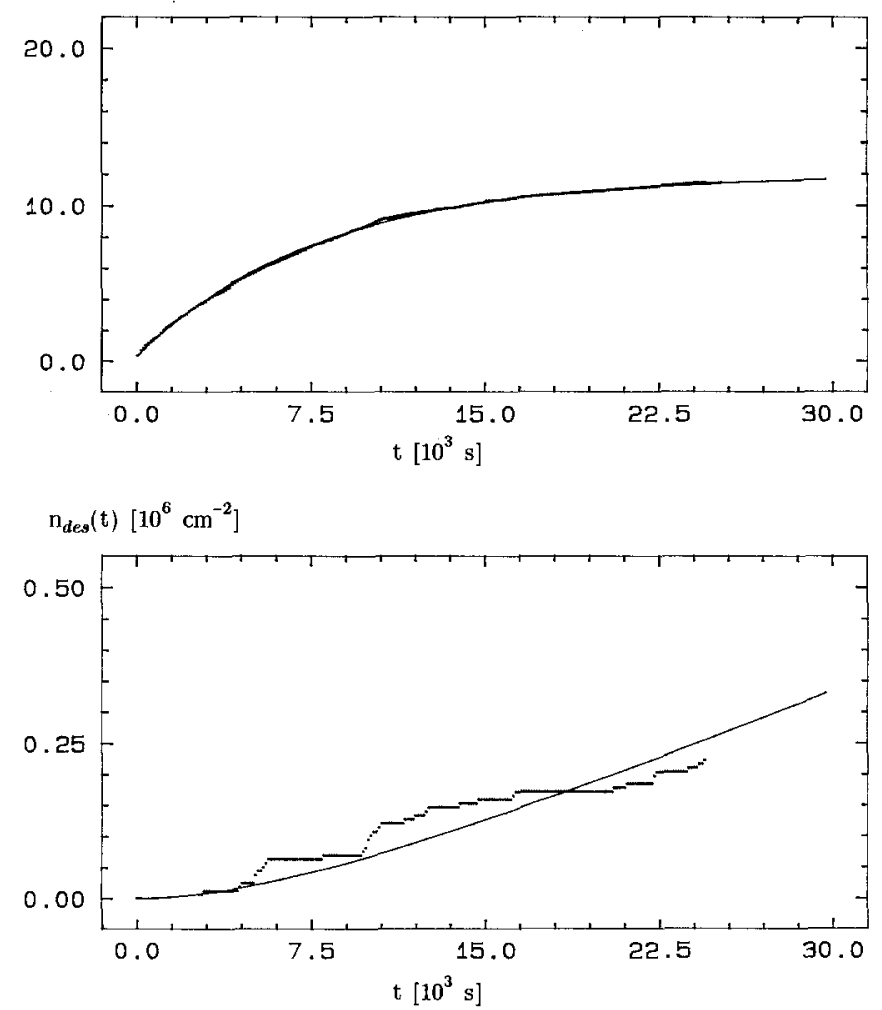

FIG. 2. Total number of deposited $n(t)$, adsorbed $n_{\mathrm{ads}}(t)$, and desorbed particles $n_{\mathrm{des}}(t)$ as a function of time for lgl30a. The drawn lines represent the best fit of Eqs. [20], [22], and [24] to the data points, respectively. 


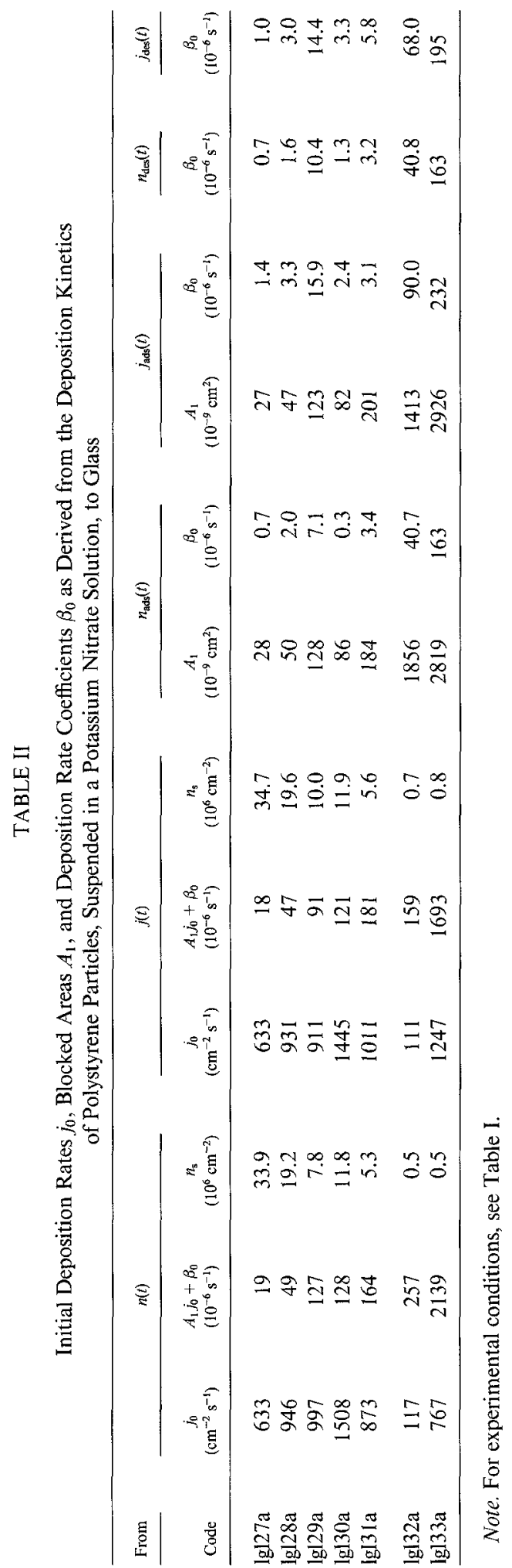

as a function of the residence time, by dividing the number of particles desorbing on time $t$ by the number of particles adsorbing on time $\tau$. Subsequently, these desorption probabilities are expressed per unit time, yielding $\beta(t-\tau)$.

If it is assumed that $\beta(t-\tau)$ is independent of residence time, it follows from Eq. [2] that comparison of the stored images can also be used directly to determine $\beta(t)$, since

$$
\beta(t)=\frac{j_{\text {des }}(t)}{n(t)}=\frac{\Delta n_{i j}}{n(t)\left(t_{j}-t_{i}\right)},
$$

where $t=\left(t_{j}+t_{i}\right) / 2$, and $\Delta n_{i j}$ is the number of particles desorbing between $t_{i}$ and $t_{j}$.

There are two obvious, potential problems involved in the above described procedure, especially with regard to the establishment of connectivity. Very seldom particles move slightly over the collector surface, which could cause the program to decide that these particles have desorbed. To avoid this, the program checks a maximum area of $7 \times 7$ pixels, i.e., one particle diameter around the center of the particle, in order to reestablish connectivity, when appropriate.

Another potential problem is that a particle can adhere on exactly the same position as from which a particle has desorbed. Because of the extremely small chances upon such an event, we decided to ignore this potential problem.

\section{Analysis of the Spatial Arrangement of Adhering Particles}

Pair distribution functions between adhering particles were calculated essentially as described by Sjollema and Busscher (30) and expressed as center-to-center distances, but with the inclusion of the times of arrival of adhering particles. This allows discrimination of upstream and downstream deposition.

Briefly, the relative local densities $g(x, y)$ of adhering particles compared to the average density were calculated by determining local densities around a central particle, while taking 


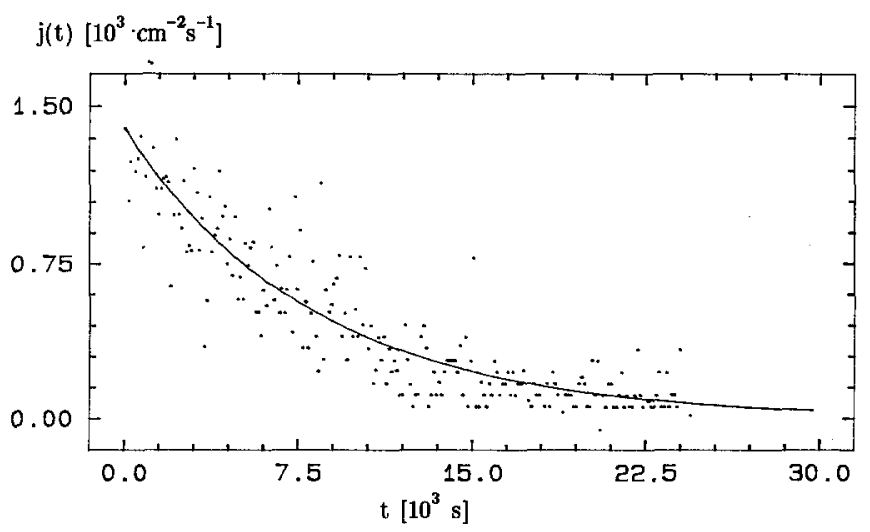

$$
\mathrm{j}_{\text {ads }}(\mathrm{t})\left[10^{3} \mathrm{~cm}^{-2} \mathrm{~s}^{-1}\right]
$$
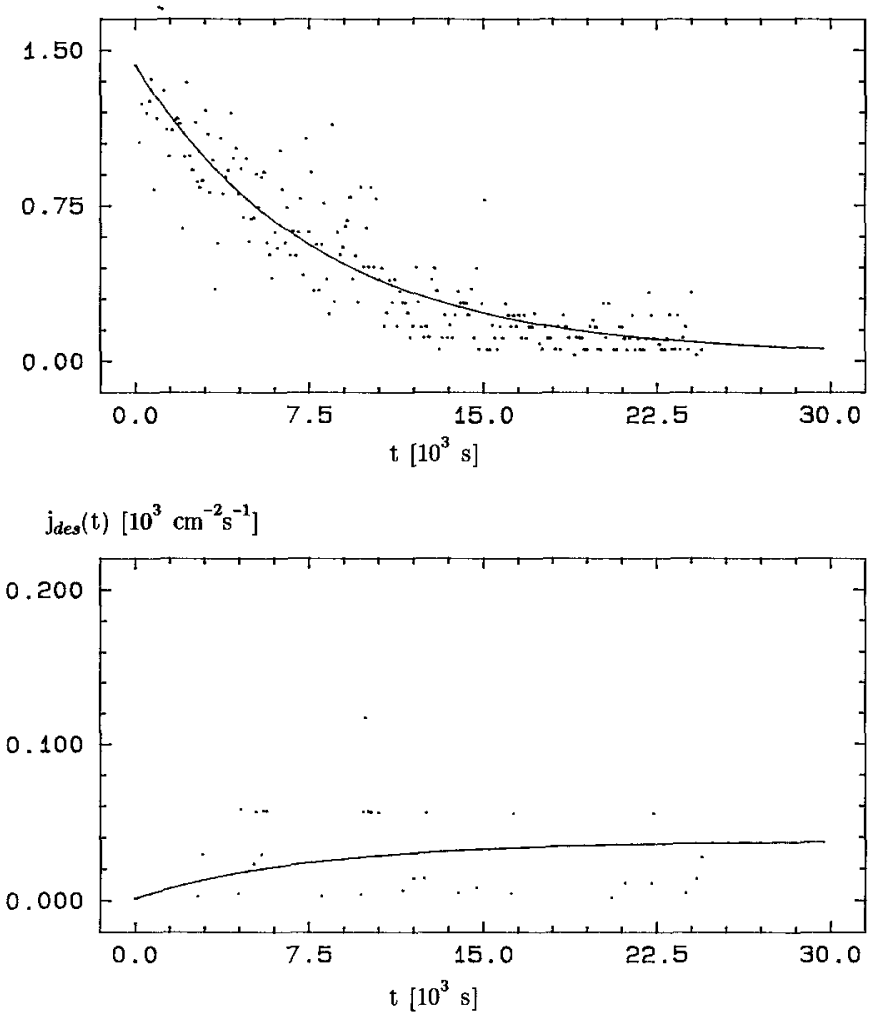

FIG. 3. Deposition $j(t)$, adsorption $j_{\text {ads }}(t)$, and desorption rate $j_{\text {des }}(t)$ as a function of time for $\lg 130 \mathrm{a}$. The drawn lines represent the best fit of Eqs. [19], [21], and [23] to the data points, respectively.

the time of adsorption into account. Subsequently each adhering particle in the image is once taken as a central particle.

Various presentation forms of $g(x, y)$ have recently been discussed by Busscher et al. (32) and include:
- a 3D presentation of the pair distribution function $g(x, y)$

- cross sections $\mathrm{g}(\mathrm{x})$ and $\mathrm{g}(\mathrm{y})$, indicating the pair distribution functions parallel and perpendicular to the direction of flow, respectively; 
- a radial pair distribution function $g(r)$, indicating the relative local density in a circular shell of thickness $d r$ and radius $r$ around the central particle. $g(r)$, however, assumes a rotational symmetry in the spatial arrangement of adhering particles, which is not necessarily present.

In order to obtain statistically reliable pair distribution functions, we have decided that an image must contain more than 1500 particles. Whenever this was not the case, images of other parts of the substratum than monitored during the experiment, were also taken and analyzed. Averaging the pair distribution functions $g(x, y)$ of each image then yields adequate statistics. In these cases we have no longer information on the time of adsorption and the distribution functions cannot dis- criminate between upstream and downstream deposition.

\section{RESULTS AND DISCUSSION}

Figure 2 presents an example of the total number of deposited, adsorbed and desorbed particles as a function of time. Using $n(t)$ and Eq. [20], the parameters $j_{0},\left(A_{1} j_{0}+\beta_{0}\right)$ and consequently $n_{\mathrm{s}}$ can be calculated with an iterative least-squares fitting procedure. All points seem to fit the theoretical curve very closely (note that the points do not appear separated due to the perfect fit). From $n_{\text {ads }}(t)$, Eq. [22], and employing $j_{0}$ and $\left(A_{1} j_{0}+\beta_{0}\right)$ derived from $n(t)$, the parameters $A_{1}$ and $\beta_{0}$ can be calculated with a similar least-squares fitting procedure. $\beta_{0}$ can also be separately calculated from $n_{\text {des }}(t)$, Eq. [24], and the data
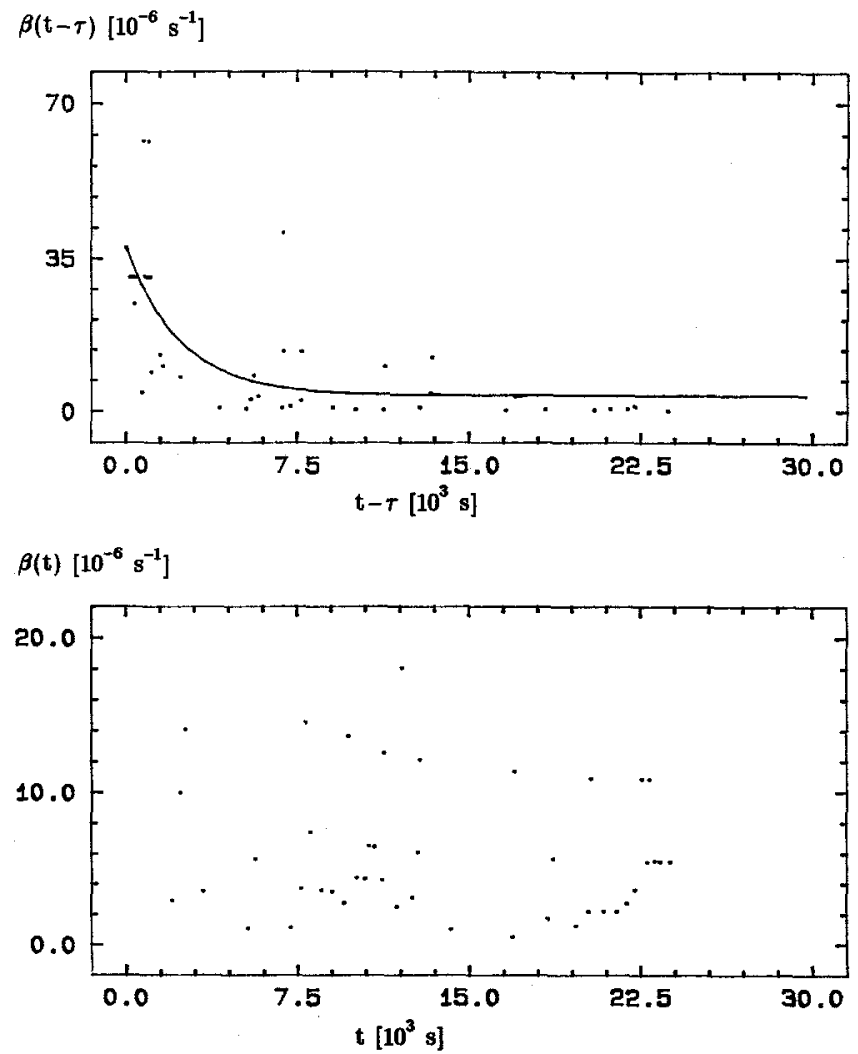

FIG. 4. Desorption rate coefficients $\beta$ as a function of the residence time (top) and as a function of time, while assuming independence of residence time (bottom) for lgl31a. The drawn line (top) represents the best fit of Eq. [3] to $\beta(t-\tau)$. 
TABLE III

Desorption Rate Coefficients as Derived from the Deposition Kinetics of Polystyrene Particles, Suspended in a Potassium Nitrate Solution, to Glass

\begin{tabular}{|c|c|c|c|c|c|}
\hline \multirow{2}{*}{$\frac{\text { From }}{\text { Code }}$} & \multicolumn{3}{|c|}{$\beta(t-\tau)$} & \multicolumn{2}{|c|}{$\beta(t)$} \\
\hline & $\begin{array}{c}\beta_{0} \\
\left(10^{-6} \mathrm{~s}^{-1}\right)\end{array}$ & $\begin{array}{c}\beta_{\infty} \\
\left(10^{-6} \mathrm{~s}^{-1}\right)\end{array}$ & $\begin{array}{c}\delta \\
\left(10^{-3} \mathrm{~s}^{-1}\right)\end{array}$ & $\begin{array}{c}\beta_{\mathrm{m}} \\
\left(10^{-6} \mathrm{~s}^{-1}\right)\end{array}$ & $\begin{array}{c}\beta_{\mathrm{g}} \\
\left(10^{-6} \mathrm{~s}^{-1}\right)\end{array}$ \\
\hline $\lg 127 a$ & 15.8 & 0.8 & 2.2 & 1.4 & 2.2 \\
\hline $\lg 128 \mathrm{a}$ & 6.8 & 0.7 & 0.3 & 3.0 & 2.9 \\
\hline $\lg 129 a$ & 91.9 & 9.3 & 1.2 & 27.6 & 6.7 \\
\hline $\operatorname{lgl} 30 \mathrm{a}$ & 6.1 & 0.5 & 0.2 & 4.0 & 3.4 \\
\hline lgl31a & 37.7 & 3.5 & 0.4 & 6.0 & 6.0 \\
\hline lgl32a & 400 & 61.6 & 2.6 & 67.5 & 70.6 \\
\hline lgl33a & 4353 & 261 & 3.0 & 195.4 & 41.0 \\
\hline
\end{tabular}

Note. Initial desorption rate coefficients $\beta_{0}$, final desorption rate coefficients $\beta_{\infty}$ and relaxation times $\delta$ determined from $\beta(t-\tau)$ and Eq. [3]; mean desorption rate coefficient $\beta_{\mathrm{m}}$ determined from $\beta(t)$, while assuming independency of the residence time; mean desorption rate coefficient $\beta_{\mathrm{g}}$ determined from $\beta(t)$ in the presence of a concentration gradient. For experimental conditions, see Table I.

derived from $n(t)$. The above data are summarized in Table II for all experiments.

Figure 3 presents an example of the rate of deposition, adsorption, and desorption as a function of time derived from a comparison of successively stored images. These data contain considerably more scatter than those in Fig. 2. This is a typical feature of all directly determined deposition, adsorption and desorption rates. Using $j(t)$ and Eq. [19], the parameters $j_{0},\left(A_{1} j_{0}+\beta_{0}\right)$, and consequently $n_{\mathrm{s}}$ can be calculated with an iterative leastsquares fitting procedure. From $j_{\mathrm{ads}}(t)$, Eq. [21], and the data derived from $n(t)$, the parameters $A_{1}$ and $\beta_{0}$ can be calculated, while from $j_{\text {des }}(t)$, Eq. [23], and the data derived from $n(t)$, the parameter $\beta_{0}$ can also be calculated. These data are also summarized for all experiments in Table II.

First from Table II it follows that there is a fairly good numerical correspondence between $j_{0}$ derived from the initial time dependence of the total counts $\mathrm{n}(\mathrm{t})$ and the $j_{0}$ derived from a comparison of successively stored images, i.e., $j(t)$. A similar remark can be made with respect to $A_{1} j_{0}+\beta_{0}$ and $n_{\mathrm{s}}$. Although it is outside the scope of this paper to look for relations between various parameters, we note that there is a marked dependence of these parameters on both flow rate and ionic strength.

Second also from Table II, it can be seen that the blocked areas $A_{1}$ derived from $n_{\text {ads }}(t)$ and $j_{\text {ads }}(t)$ correspond well, particularly at the high ionic strength. Note that the blocked areas increase with increasing flow rate, and that in some cases, the values are relatively high as compared to reported literature values (10). Possibly, this is due to a combination of real, hydrodynamic blocking and the use of relatively high ionic strengths, which could lead to a fast saturation of active centers on the collector surface.

Figure 4 gives the desorption rate coefficient $\beta$ as function of time for a selected example, derived without and with the assumption that $\beta$ is independent of the particle residence time, yielding $\beta(t-\tau)$ and $\beta(\mathrm{t})$, respectively. Using $\beta(t-\tau)$ and Eq. [3], we can derive $\beta_{0}, \beta_{\infty}$, and $\delta$ with a least-squares fitting procedure, whereas from $\beta(t)$ we have only determined a mean value $\beta_{\mathrm{m}}$. These data are summarized in Table III for all experiments. From Table III it can be seen that $\beta_{0}$ is always greater than $\beta_{\infty}$ and that $\beta_{\mathrm{m}}$ has values between $\beta_{0}$ and $\beta_{\infty}$. There is a distinct difference between $\beta_{0}$ 
presented in Table II as compared to those presented in Table III. The desorption rate coefficients, $\beta_{0}$, summarized in Table II were derived while assuming independence of the residence time, whereas those in Table III were derived while accounting for a residence time dependence. When accounting for the residence time dependence of $\beta$ (see Table III), it appears that in reality $\beta_{0}$ is higher, and that $\beta$ decreases strongly with residence time, i.e., the interaction forces become stronger in time. In fact, $\beta(t-\tau)$ obeyed Eq. [3] quite well and

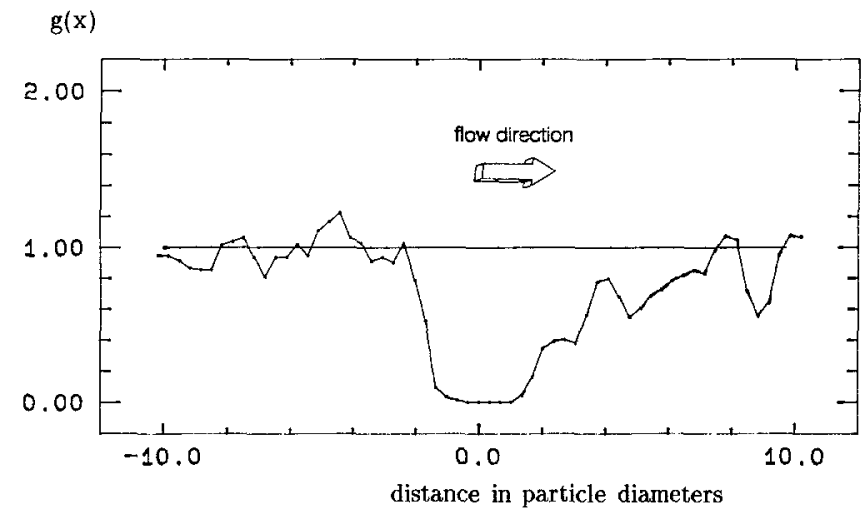

$g(y)$

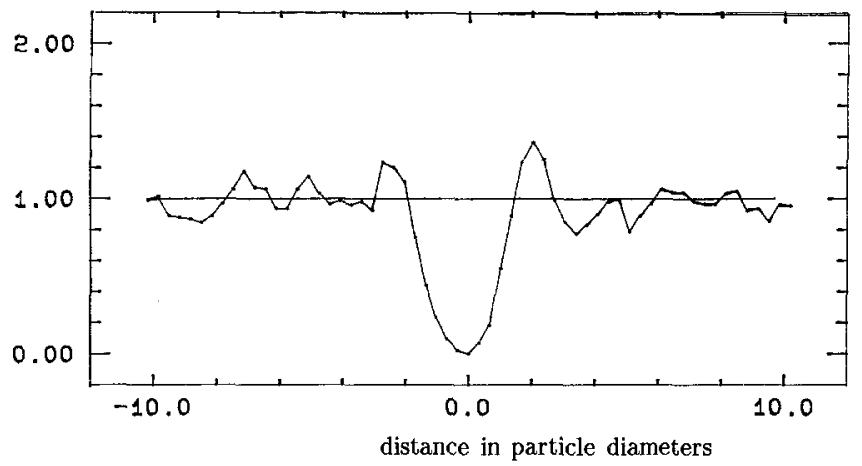

$\mathrm{g}(\mathrm{r})$

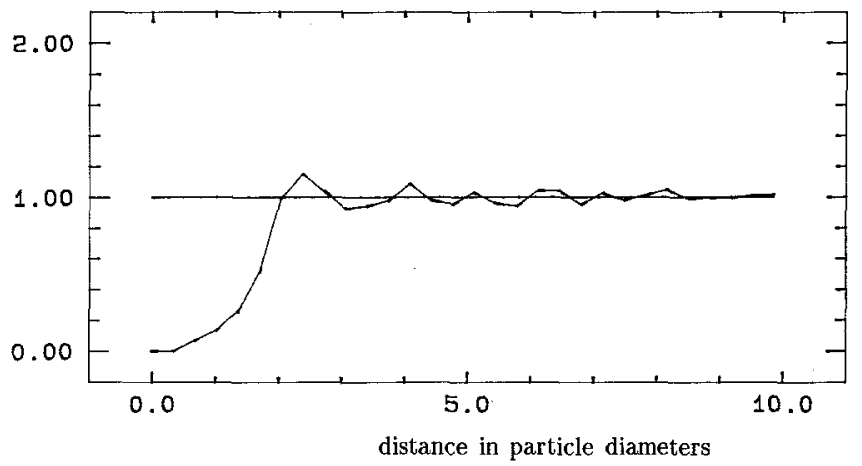

FIG. 5. Cross sections $g(x)$, parallel to the direction of flow, $g(y)$, transverse to the direction of flow, and radial pair distribution functions $g(r)$, for $\operatorname{lgl} 30 \mathrm{a}$. The arrow indicates the direction of flow; distances are expressed as center-to-center. 
a relaxation time $\delta$ could be derived, representing the characteristic time of increase of the interaction forces. This decrease in desorption probability may originate from e.g. progressive removal of interfacial water, reorientation of dipoles or particles as a whole, or an increase of the number of hydrogen bonds.

Figure 5 shows pair distribution functions for a selected example. Distinguishing between upstream and downstream deposition clearly shows a decreased probability of downstream
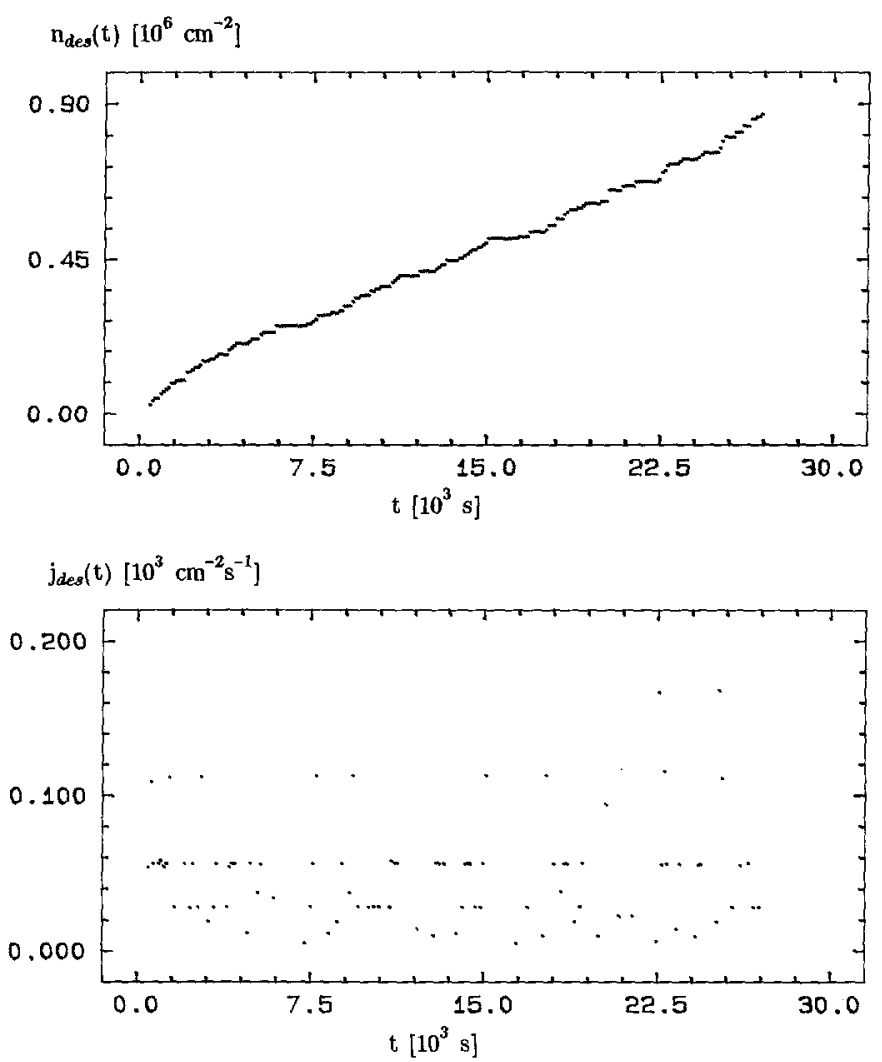

$\beta(\mathrm{t})\left[10^{-3} \mathrm{~s}^{-1}\right\}$

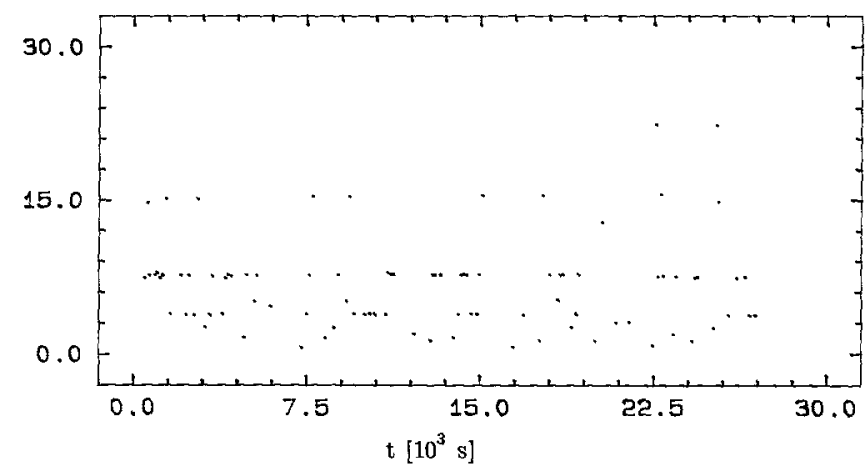

FIG. 6. Total desorbed number of particles $n_{\text {des }}(t)$, desorption rate $j_{\text {des }}(t)$, and the desorption rate coefficient $\beta(t)$ for lgl29a during desorption under influence of a concentration gradient. 
deposition, as can be seen from the asymmetrical distribution of $g(x)$ in the direction of flow. However, the pair distribution function transverse to the direction of flow, $g(y)$, is symmetrical, as expected. Because $g(r)$ assumes rotational symmetry, we conclude that $g(r)$ is, in general, not an ideal representation of pair distribution functions.

Figures 6 and 7 present examples of desorption of polystyrene particles stimulated by a concentration gradient and a surfactant, respectively. As can be seen from Fig. 6, flowing
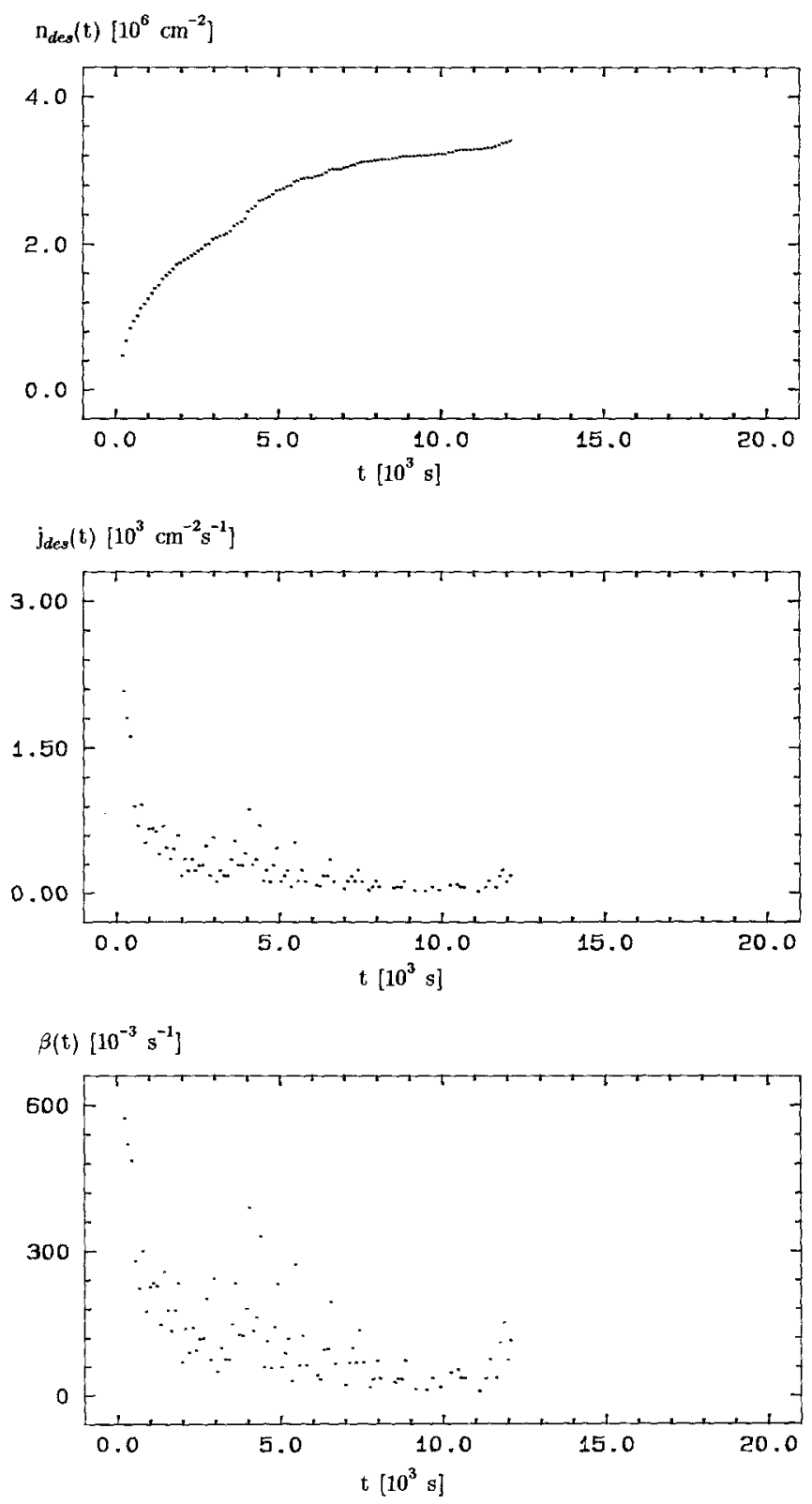

FIG. 7. Total desorbed number of particles $n_{\text {des }}(t)$, desorption rate $j_{\text {des }}(t)$, and the desorption rate coefficient $\beta(t)$ for lgl31a during desorption under influence of a surfactant. 
with a potassium nitrate solution without any particles yields minor desorption. Desorption rate coefficients, $\beta_{\mathrm{g}}$, calculated by determining the average of $\beta(t)$, varied between $2 \times 10^{-6}$ and $7 \times 10^{-6} \mathrm{~s}^{-1}$ at the high ionic strength and between $41 \times 10^{-6}$ and $70 \times 10^{-6} \mathrm{~s}^{-1}$ at the low ionic strength (see also Table III). Desorption stimulated by a surfactant was much higher (compare Figs. 6 and 7) and yielded desorption rate coefficients between $50 \times 10^{-6}$ and $300 \times 10^{-6} \mathrm{~s}^{-1}$ at the high ionic strength and between $200 \times 10^{-6}$ and $250 \times 10^{-6} \mathrm{~s}^{-1}$ at the low ionic strength.

\section{CONCLUSIONS}

In this paper we described a technique to simultaneously determine the adsorption and desorption of colloidal particles. The technique is based on the availability of a personal computer with an image analyzer. In the absence of such instrumentation particle deposition has been measured mainly in terms of total numbers of particles adhering as a function of time, i.e., $n(t)$. The method described in this paper compares successively stored images yielding simultaneously measurement of adsorption and desorption.

The establishment of connectivity of particles in successive images is crucial in the method described. Desorption can happen sometimes extremely fast as proven by semiautomatic and manual analysis of video images (35). With the personal computer used in this study, our fully automatic program cannot establish connectivity when particles are residing on a surface for less than $110 \mathrm{~s}$. This aspect can be improved by the use of faster computers and image analyzers. Another drawback is the fact that our image analyzer only has $512 \times 512$ pixels, thus relatively high magnifications have to be used in order to obtain an adequate number of pixels per particle. Image analyzers with higher pixel resolution will enable working at lower magnification, therewith increasing the area of the field of view and improving the statistics.

Despite these limitations of the system, we have observed a consistency within data derived from analysis of classical, total counts $n(t)$, and a comparison of successively stored images. Furthermore, since the program stores the time of arrival of each adhering particle, pair distribution functions can be calculated that distinguish between upstream and downstream deposition with respect to the direction of flow. Also it is emphasized that a minor desorption of adhering particles was observed both during the deposition process, as well as afterwards when flowing with buffer, indicating that principally the process of particle adhesion might be considered as a reversible one.

\section{ACKNOWLEDGMENT}

The authors are greatly indebted to Dr. Th. F. Thadros for critically commenting on the manuscript.

\section{REFERENCES}

1. Lalande, M., Tissier, J. P., and Corrieu, G., J. Dairy Res. 51, 557 (1984).

2. Adamczyk, Z., Czarnecki, J., and Warszynski, P., J. Colloid Interface Sci. 106, 299 (1985).

3. Reynolds, P. A., and Goodwin, J. W., Colloids Surf. 23, 273 (1987).

4. Van de Ven, T. G. M., J. Colloid Interface Sci. 124, 138 (1988).

5. Schulze, H. J., Wahl, B., and Gottschalk, G., J. Colloid Interface Sci. 128, (1989).

6. Weerkamp, A. H., Quirynen, M., Marechal, M., Van der Mei, H. C., Van Steenberghe, D., and Busscher, H. J., Microb. Ecol. Health Disease 2, 11 (1989).

7. Schaeken, M. J. M., Van der Hoeven, J. S., and Hendriks, J. C. M., J. Dent. Res. 68, 1786 (1989).

8. Adamczyk, Z., Colloids Surf. 35, 283 (1989).

9. Duddridge, J. E., Kent, C. A., and Laws, J. F., Biotechnol. Bioeng. XXIV, 153 (1982).

10. Dabros, T., and Van de Ven, T. G. M., Colloid Polym. Sci. 261, 694 (1983).

11. Dabros, T., and Van de Ven, T. G. M., Phys. Chem. Hydrodyn. 8, 161 (1987).

12. Prieve, D. C., and Lin, M. M. J., J. Colloid Interface Sci. 76, 32 (1980).

13. Absolom, D. R., Strong, A. B., Ledain, C., Thomson, B. E., and Zingg, W., Trans Am. Soc. Artif. Intern. Organs XXVIII, 413 (1982).

14. Sjollema, J., Busscher, H. J., and Weerkamp, A. H., Biofouling 1, 101 (1988).

15. Dabros, T., and Van de Ven, T. G. M., J. Colloid Interface Sci. 89, 232 (1982).

Journal of Colloid and Interface Science, Vol. 152, No. 1, August 1992 
16. Dabros, T., and Van de Ven, T. G. M., J. Colloid Interface Sci. 93, 576 (1983).

17. Varoqui, R., and Pefferkorn, E., J. Colloid Interface Sci. 109, 520 (1986).

18. Kallay, N., Biskup, B., Tomic, M., and Matijevic, E., $J$. Colloid Interface Sci. 114, 357 (1986).

19. Adamczyk, Z., and Petlicki, J., J. Colloid Interface Sci. 118, 20 (1987).

20. Sjollema, J., and Busscher, H. J., Colloids Surf. 47, 323 (1990).

21. Busscher, H. J., Sjollema, J., and Van der Mei, H. C., in "Microbial Cell Surface Hydrophobicity" (R. J. Doyle and M. Rosenberg, Eds.), p. 335. American Society for Microbiology, Washington, DC, 1990.

22. Korber, D. R., Lawrence, J. R., Zhang, L., and Caldwell, D. E., Biofouling 2, 335 (1990).

23. Adamczyk, Z., and Van de Ven, T. G. M., J. Colloid Interface Sci. 80, 340 (1981).

24. Adamczyk, Z., and Van de Ven, T. G. M., J. Colloid Interface Sci. 97, 68 (1984).
25. Adamczyk, Z., Dabros, T., Czarnecki, J., and Van de Ven, T. G. M., J. Colloid Interface Sci. 97, 91 (1984).

26. Peters, M. H., J. Colloid Interface Sci. 121, 179 (1988)

27. Adamczyk, Z., Colloids Surf. 39, 1 (1989).

28. Dabros, T., Colloids Surf. 39, 127 (1989).

29. Nelson, C. H., Robinson, J. A., and Characklis, W. G., Biotechnol. Bioeng. XXVII, 1662 (1985).

30. Sjollema, J., and Busscher, H. J., Colloids Surf. 47, 337 (1990).

31. Sjollema, J., Van der Mei, H. C., Uyen, H. M., and Busscher, H. J., FEMS Microbiol. Lett. 69, 263 (1990).

32. Busscher, H. J., Noordmans, J., Meinders, J. M., and Van der Mei, H. C., Biofouling, 4, 71 (1991).

33. Brouwer, W. M., and Zsom, R. L. J., Colloids Surf. 24, 195 ( 1987).

34. Sjollema, J., Busscher, H. J., and Weerkamp, A. H., J. Microbiol. Methods 9, 73 (1989).

35. Capson, D. W., Maludzinski, R. A., and Feuerstein, I. A., IEEE Trans. Biomed. Eng. 36, 860 (1989). 